\title{
Obesity and COVID-19 in adult patients with diabetes
}

Peihua Cao, Ying Song, Zian Zhuang, Jinjun Ran, Lin Xu, Yan Geng, Lefei Han, Shi Zhao, Jing Qin, Daihai He, Fengfu Wu, Lin Yang

\section{Online-only Supplemental Material}

Supplementary Table 1. Comparison of COVID-19 patients with and without BMI data.

Supplementary Table 2. Likelihood ratio tests between interaction models and multivariable models.

Supplementary Table 3. Crude and adjusted odds ratio (OR) of severe outcomes of COVID-19 of underweight, overweight and obese patients, with reference to normal weight patients. The subgroups are hypertensive patients with and without ARB prescription, and the patients with and without IL-6 inhibitor prescription in hospital..5 Supplementary Table 4. Adjusted odds ratio (OR) estimated from sensitivity analyses: 1) by using raw data, and 2) adding smoking status as covariate...............................................6 Supplementary Table 5. Sensitivity analysis for crude and adjusted odds ratio (OR) of severe outcomes of COVID-19 of underweight, overweight and obese patients, with reference to normal weight patients, from multiple imputed data. 


\section{Supplementary Table 1. Comparison of COVID-19 patients with and without BMI data.}

\begin{tabular}{|c|c|c|c|}
\hline Characteristics & $\begin{array}{l}\text { With BMI data } \\
(n=1637)\end{array}$ & $\begin{array}{l}\text { Without BMI data } \\
(n=1340)\end{array}$ & $\mathrm{P}$ \\
\hline Age, years, median [IQR] & $60 \cdot 00[50 \cdot 00,68 \cdot 00]$ & $61 \cdot 00[49 \cdot 00,69 \cdot 00]$ & $0 \cdot 335$ \\
\hline Gender, male, n, \% & $814(49 \cdot 7)$ & $703(52 \cdot 5)$ & $0 \cdot 147$ \\
\hline Smoking status, n, \% & & & $0 \cdot 181$ \\
\hline Never/unknown & $1214(92 \cdot 6)$ & $972(91 \cdot 0)$ & \\
\hline Former/current & $97(7 \cdot 4)$ & $96(9 \cdot 0)$ & \\
\hline \multicolumn{4}{|l|}{ Comorbidities n, \% } \\
\hline Any & $642(39 \cdot 2)$ & $558(41 \cdot 6)$ & $0 \cdot 192$ \\
\hline None & $995(60 \cdot 8)$ & $782(58 \cdot 4)$ & $0 \cdot 320$ \\
\hline 1 & $415(25 \cdot 4)$ & $350(26 \cdot 1)$ & \\
\hline$>1$ & $227(13 \cdot 9)$ & $208(15 \cdot 5)$ & \\
\hline Malignancy & $29(1 \cdot 8)$ & $25(1 \cdot 9)$ & $0 \cdot 181$ \\
\hline Hypertension & $473(28 \cdot 9)$ & $405(30 \cdot 2)$ & $0 \cdot 453$ \\
\hline Coronary artery disease & $90(5 \cdot 5)$ & $92(6 \cdot 9)$ & $0 \cdot 141$ \\
\hline Congestive heart failure & $16(1 \cdot 0)$ & $26(1 \cdot 9)$ & 0.039 \\
\hline COPD & $46(2 \cdot 8)$ & $53(4 \cdot 0)$ & $0 \cdot 103$ \\
\hline Chronic kidney disease & $6(0 \cdot 4)$ & $10(0 \cdot 7)$ & $0 \cdot 247$ \\
\hline Chronic liver disease & $32(2 \cdot 0)$ & $41(3 \cdot 1)$ & $0 \cdot 069$ \\
\hline Diabetes mellitus & $231(14.1)$ & $185(13.8)$ & $0 \cdot 853$ \\
\hline \multicolumn{4}{|c|}{ Symptoms on admission, n, \% } \\
\hline Fever & $854(52 \cdot 2)$ & $739(55 \cdot 1)$ & $0 \cdot 113$ \\
\hline Chills & $69(4 \cdot 2)$ & $63(4 \cdot 7)$ & $0 \cdot 581$ \\
\hline Dry cough & $760(46 \cdot 4)$ & $678(50 \cdot 6)$ & $0 \cdot 026$ \\
\hline Sore throat & $69(4 \cdot 2)$ & $48(3 \cdot 6)$ & $0 \cdot 430$ \\
\hline Myalgia & $462(28 \cdot 2)$ & $433(32 \cdot 3)$ & $0 \cdot 017$ \\
\hline Fatigue & $471(28 \cdot 8)$ & $449(33 \cdot 5)$ & $0 \cdot 006$ \\
\hline Headache & $54(3 \cdot 3)$ & $43(3 \cdot 2)$ & $0 \cdot 973$ \\
\hline Diarrhoea & $127(7 \cdot 8)$ & $98(7 \cdot 3)$ & $0 \cdot 699$ \\
\hline Dyspnoea & $355(21 \cdot 7)$ & $366(27 \cdot 3)$ & $<0 \cdot 001$ \\
\hline RR, median [IQR] & $20 \cdot 00[19 \cdot 00,22 \cdot 00]$ & $20 \cdot 00[19 \cdot 00,21 \cdot 00]$ & $0 \cdot 001$ \\
\hline $\mathrm{SBP}, \mathrm{mm} \mathrm{Hg}$, median [IQR] & $130 \cdot 00[120 \cdot 00,140 \cdot 00]$ & $129 \cdot 00[120 \cdot 00,140 \cdot 00]$ & $0 \cdot 718$ \\
\hline
\end{tabular}




$\begin{array}{llll}\text { DBP, mm Hg, median }[\mathrm{IQR}] & 80 \cdot 00[74 \cdot 00,89 \cdot 00] & 80 \cdot 00[74 \cdot 00,88 \cdot 00] & 0 \cdot 362 \\ \text { FPG, mmol, median [IQR] } & 4.96[4.55,5.80] & 5.11[4.62,6.42] & <0.001 \\ \text { CT image, n, \% } & & \\ \text { Ground-glass opacity } & 459(28 \cdot 0) & 604(45 \cdot 1) & <0.001 \\ \text { Bilateral pulmonary infiltration } & 25(1 \cdot 5) & 51(3 \cdot 8) & <0.001 \\ \text { Consolidation } & & & 0.001 \\ \quad \text { Left lung } & 46(2 \cdot 8) & 24(1 \cdot 8) & \\ \quad \text { Right lung } & 64(3 \cdot 9) & 47(3 \cdot 5) & 485(36 \cdot 2) \\ \text { Both lungs } & 686(41 \cdot 9) & \end{array}$

Abbreviations: IQR, interquartile range; COPD, Chronic obstructive pulmonary disease; RR, respiratory rate; SBP, systolic blood pressure; DBP, diastolic blood pressure; FPG, Fasting plasma glucose level

^BMI categories: underweight, BMI $<18.5 \mathrm{~kg} / \mathrm{m}^{2}$, normal weight, $18.5-23.9 \mathrm{~kg} / \mathrm{m}^{2}$, overweight, $24.0-27.9 \mathrm{~kg} / \mathrm{m}^{2}$, and obesity, $\geq 28 \mathrm{~kg} / \mathrm{m}^{2}$

\# P-value was calculated from Kruskal-Wallis test for non-normally distributed continuous variables, from Chi-square test or Fisher exact test for categorical variables. 


\section{Supplementary Table 2. Likelihood ratio tests between interaction models and multivariable models.}

\begin{tabular}{|c|c|c|c|c|c|c|}
\hline & \multicolumn{3}{|c|}{ Severe pneumonia } & \multicolumn{3}{|c|}{ Oxygen therapy } \\
\hline & $\begin{array}{c}\text { Log- } \\
\text { likelihood }\end{array}$ & $\begin{array}{l}\text { Degree of } \\
\text { Freedom }\end{array}$ & $P^{*}$ & $\begin{array}{c}\text { Log- } \\
\text { likelihood }\end{array}$ & $\begin{array}{l}\text { Degree of } \\
\text { Freedom }\end{array}$ & $P^{*}$ \\
\hline Model 1 & $-2181 \cdot 4$ & 8 & - & $-2060 \cdot 5$ & 8 & - \\
\hline Model 2 & $-2172 \cdot 2$ & 11 & $<0.001$ & $-2058 \cdot 1$ & 11 & $0 \cdot 195$ \\
\hline Model 3 & $-2176 \cdot 8$ & 11 & $<0.001$ & $-2058 \cdot 5$ & 11 & $<0.001$ \\
\hline Model 4 & $-2177 \cdot 8$ & 12 & $0 \cdot 143$ & $-2056 \cdot 5$ & 12 & $0 \cdot 049$ \\
\hline Model 5 & $-2175 \cdot 9$ & 12 & $0 \cdot 027$ & $-2056 \cdot 6$ & 12 & $0 \cdot 096$ \\
\hline Model 6 & $-2155 \cdot 3$ & 12 & $<0.001$ & $-2049 \cdot 6$ & 12 & $<0.001$ \\
\hline
\end{tabular}

Model 1 (as reference) includes covariates of BMI, age, sex, and comorbidities score;

Model 2 adds an interaction term of BMI*age to model 1;

Model 3 adds an interaction term of BMI* sex to model 1;

Model 4 adds a covariate of diabetes and an interaction term of BMI*diabetes to model 1;

Model 5 adds a covariate of ARB prescription and an interaction term of BMI* ARB prescription to model 1;

Model 6 adds a covariate of IL-6 inhibitor prescription and an interaction term of BMI* IL-6 inhibitor prescription to model 1 . 
Supplementary Table 3. Crude and adjusted odds ratio (OR) of severe outcomes of COVID-19 of underweight, overweight and obese patients, with reference to normal weight patients. The subgroups are hypertensive patients with and without ARB prescription, and the patients with and without IL-6 inhibitor prescription in hospital.

\begin{tabular}{|c|c|c|c|c|c|c|c|c|}
\hline & \multicolumn{4}{|c|}{ Severe Pneumonia } & \multicolumn{4}{|c|}{ Oxygen therapy } \\
\hline & Crude OR & $\mathrm{P}$ & Adjusted OR* & $P$ & Crude OR & $\mathrm{P}$ & Adjusted OR* & $\mathrm{P}$ \\
\hline \multicolumn{9}{|l|}{ ARB prescription } \\
\hline Underweight & $1.63(0.34,7 \cdot 79)$ & $0 \cdot 541$ & $2 \cdot 03(0 \cdot 39,10 \cdot 61)$ & $0 \cdot 404$ & $1.06(0 \cdot 22,5 \cdot 14)$ & $0 \cdot 942$ & $1 \cdot 17(0 \cdot 23,5 \cdot 98)$ & $0 \cdot 851$ \\
\hline Overweight & $1 \cdot 54(0 \cdot 78,3 \cdot 23)$ & $0 \cdot 205$ & $2.08(0.94,4.59)$ & $0 \cdot 070$ & $2 \cdot 21(1 \cdot 08,4 \cdot 54)$ & $0 \cdot 030$ & $2 \cdot 35(1 \cdot 08,5 \cdot 13)$ & $0 \cdot 031$ \\
\hline Obesity & $1 \cdot 20(0 \cdot 45,3 \cdot 17)$ & $0 \cdot 716$ & $1 \cdot 34(0 \cdot 48,3 \cdot 76)$ & $0 \cdot 577$ & $1 \cdot 15(0 \cdot 41,3 \cdot 21)$ & $0 \cdot 797$ & $1 \cdot 17(0 \cdot 41,3 \cdot 36)$ & $0 \cdot 771$ \\
\hline \multicolumn{9}{|l|}{ No ARB } \\
\hline Underweight & $0 \cdot 37(0 \cdot 15,0 \cdot 891)$ & $0 \cdot 027$ & $0.42(0 \cdot 17,1 \cdot 06)$ & $0 \cdot 066$ & $0 \cdot 96(0 \cdot 436,2 \cdot 13)$ & $0 \cdot 925$ & $1 \cdot 15(0 \cdot 51,2 \cdot 62)$ & $0 \cdot 734$ \\
\hline Overweight & $0 \cdot 82(0 \cdot 606,1 \cdot 107)$ & $0 \cdot 194$ & $0 \cdot 86(0 \cdot 63,1 \cdot 18)$ & $0 \cdot 354$ & $0.75(0.558,1.02)$ & $0 \cdot 067$ & $0 \cdot 80(0.59,1 \cdot 09)$ & $0 \cdot 15$ \\
\hline Obesity & $0.77(0.493,1.211)$ & $0 \cdot 260$ & $0 \cdot 80(0 \cdot 50,1 \cdot 27)$ & $0 \cdot 339$ & $1 \cdot 10(0 \cdot 701,1 \cdot 72)$ & $0 \cdot 686$ & $1 \cdot 12(0 \cdot 71,1 \cdot 76)$ & $0 \cdot 629$ \\
\hline \multicolumn{9}{|c|}{ Tocilizumab prescription } \\
\hline Underweight & $1 \cdot 37^{*} 10^{\wedge} 7(0$, Inf $)$ & 0.989 & $2 \cdot 92 * 10^{\wedge} 6(0, \mathrm{Inf})$ & $0 \cdot 990$ & $2 \cdot 05(0 \cdot 27,15 \cdot 57)$ & $0 \cdot 488$ & $4.47(0.33,60 \cdot 99)$ & $0 \cdot 261$ \\
\hline Overweight & $1 \cdot 44(0 \cdot 47,4 \cdot 4)$ & $0 \cdot 521$ & $1 \cdot 34(0 \cdot 40,4 \cdot 46)$ & 0.636 & $0.86(0 \cdot 32,2 \cdot 28)$ & $0 \cdot 754$ & $0 \cdot 85(0 \cdot 31,2 \cdot 34)$ & $0 \cdot 755$ \\
\hline Obesity & $0.98(0 \cdot 14,6 \cdot 85)$ & 0.982 & $1 \cdot 58(0 \cdot 19,13 \cdot 18)$ & 0.673 & $0.41(0 \cdot 08,2 \cdot 16)$ & $0 \cdot 293$ & $0.46(0.08,2 \cdot 78)$ & $0 \cdot 395$ \\
\hline \multicolumn{9}{|l|}{ No tocilizumab } \\
\hline Underweight & $0.84(0.56,1 \cdot 19)$ & $0 \cdot 321$ & $0 \cdot 83(0.58,1 \cdot 19)$ & $0 \cdot 303$ & $0.82(0.54,1 \cdot 24)$ & $0 \cdot 350$ & $0 \cdot 75(0.49,1 \cdot 14)$ & $0 \cdot 177$ \\
\hline Overweight & $1 \cdot 15(0.98,1 \cdot 34)$ & $0 \cdot 080$ & $1 \cdot 17(1 \cdot 00,1 \cdot 37)$ & $0 \cdot 049$ & $0.95(0 \cdot 80,1 \cdot 13)$ & $0 \cdot 543$ & $0.99(0.83,1 \cdot 18)$ & $0 \cdot 868$ \\
\hline Obesity & $1.50(1 \cdot 18,1 \cdot 92)$ & $0 \cdot 001$ & $1 \cdot 54(1 \cdot 20,1 \cdot 98)$ & $0 \cdot 001$ & $1 \cdot 56(1 \cdot 21,2 \cdot 02)$ & $0 \cdot 001$ & $1 \cdot 67(1 \cdot 28,2 \cdot 16)$ & $<0 \cdot 001$ \\
\hline
\end{tabular}

*Multivariable models include BMI, age, sex, and comorbidity scores. 
Supplementary Table 4. Adjusted odds ratio (OR) estimated from sensitivity analyses: 1) by using raw data, and 2) adding smoking status as covariate.

\begin{tabular}{|c|c|c|c|c|c|c|c|c|}
\hline & \multicolumn{4}{|c|}{ Severe Pneumonia } & \multicolumn{4}{|c|}{ Oxygen therapy } \\
\hline & Sensitivity $1^{\wedge}$ & $\mathrm{P}$ & Sensitivity 2\# & $\mathrm{P}$ & Sensitivity $1^{\wedge}$ & $\mathrm{P}$ & Sensitivity 2\# & $P$ \\
\hline \multicolumn{9}{|l|}{ All patients } \\
\hline Underweight & $0.97(0.57,1.63)$ & $0 \cdot 896$ & $0.81(0.55,1 \cdot 20)$ & $0 \cdot 292$ & $0.79(0.44,1.39)$ & $0 \cdot 410$ & $0.71(0 \cdot 48,1 \cdot 05)$ & 0.085 \\
\hline Overweight & $1 \cdot 08(0 \cdot 85,1 \cdot 37)$ & $0 \cdot 551$ & $1 \cdot 10(0 \cdot 93,1 \cdot 31)$ & $0 \cdot 270$ & $0.94(0.73,1.22)$ & 0.657 & $0 \cdot 96(0 \cdot 81,1 \cdot 14)$ & 0.647 \\
\hline Obesity & $1.34(0.92,1.97)$ & $0 \cdot 132$ & $1.41(1.07,1.86)$ & $0 \cdot 015$ & $1.37(0 \cdot 92,2 \cdot 03)$ & $0 \cdot 122$ & $1.36(1.04,1.79)$ & 0.025 \\
\hline \multicolumn{9}{|l|}{ Age $<65$} \\
\hline Underweight & $0.50(0 \cdot 17,1 \cdot 48)$ & $0 \cdot 211$ & $0.32(0 \cdot 14,0 \cdot 70)$ & 0.005 & $0.57(0 \cdot 20,1 \cdot 67)$ & $0 \cdot 308$ & $0.91(0.52,1.62)$ & 0.756 \\
\hline Overweight & $1.20(0.89,1.64)$ & $0 \cdot 233$ & $1.34(1.08,1.66)$ & $0 \cdot 007$ & $0.95(0 \cdot 69,1 \cdot 33)$ & $0 \cdot 776$ & $0.97(0.79,1 \cdot 20)$ & 0.799 \\
\hline Obesity & $1.08(0.66,1.76)$ & 0.764 & $1.31(0.95,1 \cdot 81)$ & $0 \cdot 098$ & $1 \cdot 17(0 \cdot 71,1 \cdot 93)$ & $0 \cdot 536$ & $1.47(1.07,2 \cdot 03)$ & 0.017 \\
\hline \multicolumn{9}{|l|}{$\mathrm{Age} \geq 65$} \\
\hline Underweight & $1 \cdot 28(0 \cdot 67,2 \cdot 45)$ & $0 \cdot 461$ & $1 \cdot 17(0.71,1.92)$ & 0.543 & $0.92(0.46,1.87)$ & $0 \cdot 825$ & $0.55(0.32,0 \cdot 95)$ & 0.033 \\
\hline Overweight & $0.90(0 \cdot 62,1 \cdot 32)$ & $0 \cdot 601$ & $0.72(0.53,0.98)$ & $0 \cdot 034$ & $0.95(0.63,1.43)$ & $0 \cdot 812$ & $0.94(0 \cdot 70,1 \cdot 27)$ & 0.681 \\
\hline
\end{tabular}




\begin{tabular}{|c|c|c|c|c|c|c|c|c|}
\hline Obesity & $2 \cdot 23(1 \cdot 12,4 \cdot 43)$ & $0 \cdot 022$ & $1.97(1 \cdot 12,3 \cdot 46)$ & $0 \cdot 019$ & $1 \cdot 87(0 \cdot 96,3 \cdot 65)$ & $0 \cdot 067$ & $1 \cdot 16(0 \cdot 68,1 \cdot 98)$ & 0.591 \\
\hline \multicolumn{9}{|l|}{ Men } \\
\hline Underweight & $1 \cdot 30(0 \cdot 62,2 \cdot 70)$ & $0 \cdot 488$ & $0.97(0.55,1.69)$ & 0.907 & $0.94(0.43,2.06)$ & $0 \cdot 879$ & $0.88(0.51,1.53)$ & 0.659 \\
\hline Overweight & $1 \cdot 17(0.84,1.62)$ & $0 \cdot 356$ & $1.23(0.97,1.55)$ & 0.094 & $0.85(0 \cdot 60,1 \cdot 20)$ & 0.354 & $0.90(0 \cdot 71,1 \cdot 14)$ & $0 \cdot 365$ \\
\hline Obesity & $1.54(0.90,2 \cdot 65)$ & $0 \cdot 116$ & $1.79(1.23,2.62)$ & 0.003 & $1 \cdot 21(0 \cdot 69,2 \cdot 14)$ & 0.502 & $1 \cdot 30(0.89,1.90)$ & $0 \cdot 170$ \\
\hline \multicolumn{9}{|l|}{ Women } \\
\hline Underweight & $0.74(0.34,1.58)$ & 0.432 & $0 \cdot 70(0 \cdot 40,1 \cdot 22)$ & $0 \cdot 209$ & $0.66(0.28,1.55)$ & 0.338 & $0 \cdot 58(0 \cdot 34,1 \cdot 01)$ & 0.054 \\
\hline Overweight & $1.00(0 \cdot 70,1 \cdot 41)$ & $0 \cdot 979$ & $1 \cdot 00(0 \cdot 78,1 \cdot 28)$ & 0.985 & $1.07(0.74,1.54)$ & 0.740 & $1.00(0.77,1.28)$ & 0.980 \\
\hline Obesity & $1 \cdot 18(0 \cdot 68,2 \cdot 03)$ & 0.563 & $1.03(0.68,1.57)$ & $0 \cdot 881$ & $1.49(0 \cdot 86,2 \cdot 60)$ & $0 \cdot 159$ & $1 \cdot 34(0 \cdot 90,2 \cdot 00)$ & $0 \cdot 151$ \\
\hline \multicolumn{9}{|l|}{ Diabetes } \\
\hline Underweight & $1.45(0 \cdot 39,5 \cdot 48)$ & $0 \cdot 580$ & $1 \cdot 39(0 \cdot 56,3 \cdot 44)$ & $0 \cdot 482$ & $1 \cdot 19(0 \cdot 28,5 \cdot 05)$ & $0 \cdot 816$ & $1 \cdot 06(0.42,2 \cdot 68)$ & 0.906 \\
\hline Overweight & $0.91(0.49,1.66)$ & 0.748 & $0 \cdot 81(0 \cdot 51,1 \cdot 29)$ & $0 \cdot 370$ & $1 \cdot 66(0 \cdot 86,3 \cdot 19)$ & $0 \cdot 132$ & $1.57(0.99,2.48)$ & 0.057 \\
\hline Obesity & $1.02(0 \cdot 40,2 \cdot 56)$ & 0.975 & $1.53(0 \cdot 72,3 \cdot 25)$ & $0 \cdot 270$ & $2 \cdot 34(0 \cdot 89,6 \cdot 16)$ & 0.085 & $1 \cdot 54(0 \cdot 73,3 \cdot 28)$ & $0 \cdot 261$ \\
\hline
\end{tabular}

^: Adjusted for age, sex, and comorbidity score; \#: Adjusted for age, sex, comorbidity score and smoking status.

Supplementary Table 5. Sensitivity analysis for crude and adjusted odds ratio (OR) of severe outcomes of COVID-19 of underweight, overweight and obese patients, with reference to normal weight patients, from multiple imputed data.

\begin{tabular}{|c|c|c|c|c|c|c|c|c|}
\hline & \multicolumn{4}{|c|}{ Severe Pneumonia } & \multicolumn{4}{|c|}{ Oxygen therapy } \\
\hline & \multicolumn{2}{|c|}{ Crude OR } & \multicolumn{2}{|c|}{ Adjusted OR } & \multicolumn{2}{|c|}{ Crude OR } & \multicolumn{2}{|c|}{ Adjusted OR } \\
\hline & Before MI & After MI & Before MI & After MI & Before MI & After MI & Before MI & After MI \\
\hline \multicolumn{9}{|l|}{ All patients } \\
\hline Underweight & $0.95(0.68,1.33)$ & $0.84(0.60,1.18)$ & $0.95(0.67,1.33)$ & $0.84(0.60,1.18)$ & $0.84(0.60,1.18)$ & $1.18(0.87,1.61)$ & $0.84(0.60,1.18)$ & $1.19(0.87,1.61)$ \\
\hline Overweight & $1.13(0.97,1.32)$ & $1.09(0.91,1.30)$ & $1.14(0.98,1.32)$ & $1.10(0.91,1.31)$ & $0.95(0.82,1.10)$ & $0.84(0.71,1.01)$ & $0.95(0.81,1.10)$ & $0.84(0.70,1.00)$ \\
\hline
\end{tabular}




\begin{tabular}{|c|c|c|c|c|c|c|c|c|}
\hline Obesity & $1.46(1.14,1.86)^{\#}$ & $1.28(1.01,1.62)^{\#}$ & $1.47(1.15,1.88)^{\#}$ & $1.29(1.02,1.65)^{\#}$ & $1.40(1.10,1.79)^{\#}$ & $1.21(0.96,1.53)$ & $1.40(1.10,1.79)^{\#}$ & $1.20(0.94,1.52)$ \\
\hline \multicolumn{9}{|l|}{ Age $<65$} \\
\hline Underweight & $0.54(0.30,0.97)^{\#}$ & $0.63(0.37,1.06)$ & $0.54(0.30,0.97)^{\#}$ & $0.61(0.36,1.03)$ & $0.73(0.43,1.23)$ & $1.19(0.77,1.85)$ & $0.71(0.42,1.21)$ & $1.20(0.77,1.86)$ \\
\hline Overweight & $1.26(1.05,1.52)^{\#}$ & $1.19(0.95,1.48)$ & $1.28(1.06,1.55)^{\#}$ & $1.21(0.97,1.52)$ & $0.96(0.80,1.15)$ & $0.91(0.73,1.14)$ & $0.97(0.80,1.17)$ & $0.91(0.72,1.14)$ \\
\hline Obesity & $1.26(0.95,1.69)$ & $1.16(0.87,1.54)$ & $1.28(0.95,1.71)$ & $1.19(0.88,1.60)$ & $1.23(0.92,1.64)$ & $1.24(0.94,1.65)$ & $1.25(0.93,1.67)$ & $1.23(0.92,1.64)$ \\
\hline \multicolumn{9}{|l|}{ Age $\geq 65$} \\
\hline Underweight & $1.23(0.79,1.92)$ & $1.05(0.67,1.65)$ & $1.28(0.82,2.00)$ & $1.04(0.66,1.64)$ & $0.93(0.59,1.46)$ & $1.15(0.74,1.77)$ & $0.94(0.59,1.48)$ & $1.14(0.73,1.76)$ \\
\hline Overweight & $0.92(0.71,1.19)$ & $0.92(0.68,1.25)$ & $0.90(0.69,1.17)$ & $0.92(0.67,1.25)$ & $0.93(0.72,1.21)$ & $0.73(0.54,0.99)^{\#}$ & $0.93(0.71,1.21)$ & $0.73(0.54,0.99)^{\#}$ \\
\hline Obesity & $2.28(1.41,3.67)^{\#}$ & $1.65(1.09,2.49)^{\#}$ & $2.21(1.37,3.57)^{\#}$ & $1.64(1.07,2.49)^{\#}$ & $1.93(1.22,3.04)^{\#}$ & $1.16(0.76,1.78)$ & $1.88(1.19,2.97)^{\#}$ & $1.15(0.75,1.78)$ \\
\hline \multicolumn{9}{|l|}{ Men } \\
\hline Underweight & $1.40(0.88,2.24)$ & $1.23(0.72,2.10)$ & $1.43(0.89,2.29)$ & $1.25(0.73,2.14)$ & $0.99(0.62,1.60)$ & $0.87(0.50,1.51)$ & $1.03(0.64,1.68)$ & $0.87(0.50,1.52)$ \\
\hline Overweight & $1.22(0.98,1.50)$ & $1.11(0.86,1.43)$ & $1.23(0.99,1.52)$ & $1.12(0.86,1.44)$ & $0.84(0.68,1.04)$ & $0.82(0.64,1.04)$ & $0.85(0.68,1.05)$ & $0.81(0.63,1.04)$ \\
\hline Obesity & $1.84(1.31,2.58)$ & $1.26(0.94,1.71)$ & $1.85(1.31,2.61)^{\#}$ & $1.26(0.93,1.72)$ & $1.36(0.97,1.90)$ & $1.26(0.93,1.70)$ & $1.33(0.94,1.87)$ & $1.24(0.91,1.68)$ \\
\hline \multicolumn{9}{|l|}{ Women } \\
\hline Underweight & $0.62(0.37,1.03)$ & $0.67(0.43,1.04)$ & $0.62(0.37,1.03)$ & $0.67(0.43,1.04)$ & $0.69(0.42,1.12)$ & $1.37(0.94,1.99)$ & $0.69(0.42,1.13)$ & $1.36(0.94,1.98)$ \\
\hline Overweight & $1.08(0.87,1.34)$ & $1.09(0.84,1.41)$ & $1.08(0.86,1.34)$ & $1.08(0.83,1.41)$ & $1.08(0.87,1.35)$ & $0.86(0.66,1.13)$ & $1.07(0.86,1.33)$ & $0.86(0.66,1.12)$ \\
\hline Obesity & $1.14(0.80,1.63)$ & $1.42(0.94,2.14)$ & $1.14(0.80,1.64)$ & $1.42(0.94,2.15)$ & $1.43(1.01,2.02) \#$ & $1.07(0.72,1.59)$ & $1.40(0.98,1.99)$ & $1.06(0.71,1.58)$ \\
\hline \multicolumn{9}{|l|}{ Diabetes } \\
\hline Underweight & $1.46(0.60,3.55)$ & $1.65(0.71,3.85)$ & $1.50(0.61,3.65)$ & $1.67(0.71,3.93)$ & $1.20(0.48,2.96)$ & $1.62(0.73,3.60)$ & $1.22(0.49,3.05)$ & $1.57(0.70,3.50)$ \\
\hline Overweight & $0.90(0.60,1.35)$ & $0.91(0.56,1.49)$ & $0.88(0.58,1.34)$ & $0.90(0.55,1.49)$ & $1.64(1.10,2.44)^{\#}$ & $0.88(0.55,1.41)$ & $1.68(1.11,2.54)^{\#}$ & $0.91(0.56,1.47)$ \\
\hline Obesity & $1.03(0.56,1.89)$ & $1.11(0.60,2.04)$ & $1.01(0.54,1.89)$ & $1.10(0.59,2.05)$ & $1.95(1.04,3.64)^{\#}$ & $1.36(0.72,2.57)$ & $2.06(1.08,3.92)^{\#}$ & $1.43(0.74,2.75)$ \\
\hline
\end{tabular}

Abbreviations: MI, multiple imputation; Multivariable models include BMI, age, sex, and comorbidity scores.\#: $\mathrm{P}<0.05$. 
